\title{
Oferta do teste sorológico para Hepatite B durante o pré-natal: a vivência das puérperas
}

\author{
Serological test for Hepatitis B offered during antenatal care: the women's experience \\ Oferta de prueba serológica para Hepatitis B durante el prenatal: la vivencia de las puérperas
}

\author{
Cristiane Ferraz da Silva'; Carla Luzia França AraújoII ; Marcela Matvijc de AraújoIII
}

\begin{abstract}
RESUMO: Com a finalidade de reduzir os níveis de infecção pela transmissão vertical da Hepatite B, é necessário acompanhamento mais eficaz durante pré-natal. Objetivou-se identificar como ocorreu a oferta do teste sorológico para a Hepatite B durante o pré-natal, segundo as puérperas. A pesquisa é quantiqualitiva e utilizou-se discurso do sujeito coletivo. Foi realizada no alojamento conjunto em uma maternidade escola no Rio de Janeiro. Foram entrevistadas 41 puérperas de janeiro a março de 2011. Quando questionadas sobre oferta do teste, a maioria respondeu que foi solicitado junto com os exames de rotina do pré-natal, o profissional de saúde apenas leu o resultado. Mesmo havendo recomendação do Ministério da Saúde para oferta e realização do teste sorológico para Hepatite B, há um déficit na forma como é abordado o tema com a mulher.
\end{abstract} Palavras-Chave: Transmissão vertical; Hepatite B; pré-natal; enfermagem

\begin{abstract}
In order to reduce B Hepatitis vertical transmission, more effective antenatal care is necessary. Objectives: to identify how, from the women's standpoint, the serologic test for Hepatitis B was offered during antenatal care. This quantiqualitative study using collective subject discourse was conducted at the rooming-in ward of a maternity hospital school in Rio de Janeiro, Brazil. Between January and March 2011, 41 puerperal women were interviewed. When asked how the test was offered, most answered that it was requested together with the other routine antenatal tests; health professionals merely read the result. Despite the Ministry of Health recommendation that Hepatitis B testing be offered and performed, therre shortfall in the way the subject is addressd treated with women about to give birth.

Keywords: Vertical transmission; Hepatitis B; prenatal; nursing

RESUMEN: Con el fin de reducir los niveles de infección por la transmisión vertical de la Hepatitis B, es necesario acompañamiento más eficaz durante el prenatal. Se procuró identificar como se produjo la oferta de la prueba serológica para Hepatitis B durante el prenatal, según las puérperas. La investigación es cuantitativa y se empleó discurso del sujeto colectivo. Se realizó en el alojamiento conjunto en una maternidad escuela en Río de Janeiro. Se entrevistaron 41 puérperas entre enero y marzo de 2011. Cuando interrogadas sobre la oferta de la prueba, la mayoría respondió que fue solicitado junto a las pruebas de rutina del prenatal, el profesional sanitario apenas leyó el resultado. Incluso habiendo recomendación del Ministerio de Sanidad para oferta y realización de la prueba serológica para Hepatitis B, hay un déficit en la forma de abordaje del tema con la mujer. Palabras Clave: Transmisión vertical; Hepatitis B; prenatal; enfermería.
\end{abstract}

\section{INTRODUÇÃO}

As hepatites virais são doenças conhecidas pelos profissionais de saúde. Por ter uma incidência elevada e alto custo de diagnóstico e tratamento é considerada como tema relevante de pesquisa no cenário mundial, sendo atualmente um problema de saúde pública.

A média nacional para casos de Hepatite B no Brasil alcançou 6,9\% em 2010, sendo que a região Sudeste concentrou $36,6 \%$ dos casos segundo boletim epidemiológico em $2012^{1}$.

O Ministério da Saúde recomenda a triagem sorológica para algumas doenças durante o pré-natal. A sorologia para a Hepatite B, HIV, sífilis e toxoplasmose são exemplos destas. Sobre a Hepatite B preconiza-se realizar o teste $\mathrm{HBs} \mathrm{Ag}$ em todas as gestante no terceiro trimestre da gravidez a fim de prevenir por meio da imunização (passiva e ativa) os neonatos de mães com o teste positivo no pré-natal. O diagnóstico materno para o vírus da Hepatite B (VHB) é fundamental para possibilidade de imunização dos recém-nascidos, assim prevenindo a transmissão vertical da doença ${ }^{2}$.

Destaca-se como de importância relevante o VHB por sua distribuição geográfica larga e grande

IEnfermeira. Especialista em Terapia Intensiva e Clínica Médica e Cirúrgica Geral. Responsável Técnica do Centro Municipal de Saúde Salles Netto. Rio de Janeiro, Brasil.E-mail: cris fs@yahoo.com.br.

IIDoutora em Saúde Coletiva. Professora Associada. Universidade Federal do Rio de Janeiro. Escola de Enfermagem Anna Nery. Departamento maternoinfantil. Rio de Janeiro, Brasil. E-mail: araujo.ufrj@gmail.com.

IIIEnfermeira. Especialista em Saúde da Família. Escola Nacional de Saúde Pública. Fiocruz. Rio de Janeiro, Brasil. E-mail: enf.araujo@yahoo.com.br. 
potencial de causar uma doença crônica, como a cirrose e o carcinoma hepatocelular ${ }^{3}$. Estima-se que existam no mundo cerca de 2 bilhões de indivíduos infectados pelo $\mathrm{VHB}^{4}$.

Na exposição perinatal, a transmissão mãe/filho do VHB pode ocorrer durante o parto, pela exposição do recém-nascido a sangue ou líquido aminiótico (onde está presente o VHB), durante a passagem do canal vaginal, com o contato com secreções, pela amamentação e também, raramente, por transmissão via transplacentária.

A infecção em neonatos apresenta uma taxa de cronificação muito superior àquela que encontramos na infecção do adulto, com cerca de $90 \%$ dos neonatos evoluindo para a forma crônica e podendo, no futuro, apresentar cirrose e/ou carcinoma hepatocelular, diferentemente de indivíduos que adquirem a doença ao longo da vida com aproximadamente $30 \%$ de chance de desenvolver Hepatite B crônica ${ }^{5}$.

Na consulta de enfermagem às gestantes, a participação ativa da utente através da interação com o profissional enfermeiro é fundamental par que haja troca de saberes e informações visando à promoção do autocuidado. Nessa perspectiva, através da consulta de enfermagem como um momento de diálogo, entre enfermeiro/cliente pode-se definir metas e objetivos a serem atingidos visando à melhoria nas condições de saúde do binômio mãe e filho.

O enfermeiro tem papel fundamental no acompanhamento da gestante na assistência pré-natal. As doenças sexualmente transmissíveis (DST) nas mulheres grávidas são de difícil diagnóstico devido à ausência de sintomas clínicos. A possibilidade da gestante vir a ter uma DST poderá gerar repercussões negativas para o processo gestatório. Destaca-se a importância de aprofundar a abordagem das DST em gestantes, porque dos problemas de saúde associados à prática sexual, as mulheres também tem sido as mais afetadas ${ }^{6}$.

Com a finalidade de reduzir os níveis de infecção do VHB pela transmissão vertical (mãe/filho), se vê necessário um acompanhamento mais eficaz durante o pré-natal. No Brasil, há poucos estudos de rastreamento de Hepatite B em gestantes, mesmo sendo preconizado pelo Ministério da Saúde o exame para detecção precoce da Hepatite B dentro dos exames de rotina do pré-natal em serviços públicos, ainda é banalizado?

Estudos sobre a transmissão vertical da Hepatite B são raros no Brasil, pois este exame começou a compor a rotina do pré-natal a partir de 2005, quando o marcador na infecção HBsAg passou a ser recomendado pelo Ministério da Saúde 8 .

A pesquisa em tela tem como objeto a oferta do teste sorológico para a Hepatite B durante o pré-natal.

O estímulo a estudar esta temática foi a preocupação com a prevenção da transmissão vertical da Hepatite B, frente a recomendação da realização do ex- ame de rastreamento e as possíveis consequências para a saúde materna e infantil. Dessa forma percebemos que poderíamos então potencializar o processo de assistência, prevenção e promoção da saúde contribuindo para o aumento da qualidade de vida da mãe e seu filho.

Devido os valores de persistência da prevalência da infecção da Hepatite B entre a população, é importante realizar a sorologia de triagem no pré-natal, com intuito de iniciar o tratamento precocemente e evitar a transmissão vertical ${ }^{9}$.

A presente pesquisa objetivou, identificar como ocorreu a oferta da realização do teste sorológico para a Hepatite B durante o pré-natal, segundo as puérperas.

\section{REVISÃo DE LITERATURA}

Cabe à enfermeira realizar consulta de enfermagem e prescrição da assistência de enfermagem; como integrante da equipe de saúde: prescrever medicamentos, desde que estabelecidos em programas de saúde pública e em rotina aprovada pela instituição de saúde; oferecer assistência de enfermagem à gestante, parturiente e puérpera e realizar atividades de educação em saúde ${ }^{10}$.

A realização de ações educativas no decorrer de todas as etapas do ciclo grávido-puerperal é muito importante, mas é no pré-natal que a mulher deverá ser orientada para que possa viver o parto de forma positiva, ter menos riscos de complicações no puerpério e mais sucesso na amamentação. Considerando o pré-natal e o nascimento como momentos únicos para cada mulher e uma experiência especial no universo feminino, os profissionais de saúde devem assumir a postura de educadores que compartilham saberes, buscando devolver à mulher sua autoconfiança para viver a gestação, o parto e o puerpério.

Percebe-se que quando a gestante é sensibilizada, ela poderá cuidar melhor da sua saúde, desta forma contribuir com o profissional para melhora da qualidade do atendimento prestado ${ }^{11}$.

É relevante citar que atualmente no Brasil, os estudos de prevalência para o VHB são escassos em quantidade e a maioria das pesquisas delimitadas a algum grupo populacional específico. Sendo o comportamento individual determinante da vulnerabilidade à infecção pelo $\mathrm{VHB}^{12}$.

Destaca-se que no Brasil observam-se avanços na cobertura da assistência pré-natal, porém a qualidade dessa atenção ainda não é ideal ${ }^{13}$. Com relação ao exame sorológico para a Hepatite $\mathrm{B}$, como consta na rotina pré-natal apenas como recomendação, fica a cargo do profissional e serviço de saúde onde está sendo realizado o pré-natal.

A prática do aconselhamento é ferramenta fundamental para a interação entre as subjetividades, podendo ser recíproca a troca de saberes conhecidos e os sentimentos. Assim pode-se afirmar que o aconsel- 
hamento no decorrer do pré-natal é um instrumento que possibilita quebrar a cadeia de transmissão das infecções sexualmente transmissíveis, pois permite que a gestante tenha um pensamento reflexivo sobre os riscos de infecção e a importância de se prevenir tais doenças ${ }^{14}$.

Existem medidas preventivas fundamentais no que concerne o planejamento de estratégias pautadas em reduzir as taxas de mortalidade entre a população de menores de um ano de idade. Tais medidas poderão ser obtidas através de informações sobre as condições biológicas do recém-nascido no transcorrer de uma gestação, da qualidade da assistência pré-natal e do momento do parto, como também das causas dos óbitos e a forma de evitá-los ${ }^{15}$.

\section{Metodologia}

A pesquisa é do tipo descritivo-exploratória, com abordagem quantiqualitavia. $O$ estudo foi realizado no setor de alojamento conjunto de uma maternidade escola (ME) no Município do Rio de Janeiro. Os sujeitos da pesquisa foram puérperas que recebido pela assistência pré-natal em unidades de saúde no município do Rio de Janeiro.

Foram excluídas da pesquisa, as mães que eram soropositivas para o Vírus da Imunodeficiência Humana (HIV), que se encontravam no quadro de abortamento e/ou fizeram pré-natal em outro município.

Os dados foram coletados através de entrevista semiestruturada. $\mathrm{O}$ instrumento compreendeu o perfil da puérpera, a oferta do teste sorológico para a Hepatite B durante o pré-natal e o conhecimento acerca do exame sorológico para a Hepatite B. As entrevistas foram realizadas com 41 puérperas que se encontravam no local do estudo e se disponibilizaram em participar da pesquisa. Essa coleta aconteceu no período de Janeiro a Março de 2011 no alojamento conjunto. As entrevistas somente foram realizadas após a leitura e assinatura do termo de consentimento livre e esclarecido (TCLE), foram gravadas e posteriormente transcritas.

Para análise de dados foi utilizado o Software Qualiquantisoft $\AA$ onde as entrevistas após serem transcritas foram registradas neste programa. Os dados quantitativos foram submetidos à análise estatística com cálculos de frequência absoluta e percentual. Os dados qualitativos foram separados por categorias temáticas baseadas em expressões-chaves, ideias centrais e construídos os discursos do sujeito coletivo (DSC). Os DSC foram numerados de 1 a 5 . Os dados qualitativos foram tratados conforme o referencial da análise temática e do referencial conceitual relacionado à oferta do teste sorológico e os aspectos da prevenção da transmissão vertical da hepatite $B$, incluindo o conhecimento das puérperas.
O presente estudo foi aprovado pelo Comitê de Ética em Pesquisa (CEP) da Instituição, protocolo de pesquisa CEP/ME - Universidade Federal do Rio de Janeiro - Número 15/2010, CAAE: 0015.0.361.000-10.

\section{Resultados e Discussão}

No total foram entrevistadas 41 puérperas. Dividindo-se por faixa etária, 13 (31,7\%) tinham entre 19-23 anos, 10 (24,4\%) tinham 24-28 e com o mesmo valor também as faixas etárias entre 29-33 anos. Com menor representatividade, 5 (12,2\%) tinham entre 34-38 anos e 3 (7,3\%) entre 39-43.

Analisando os dados encontrados quanto à realização do pré-natal na gestação, 40 (97,6\%) puérperas estiveram presentes neste serviço, apenas $1(2,4 \%)$ não foi às consultas de pré-natal. $\bigcirc$ grupo de mulheres entrevistadas inclui-se, quase em sua totalidade, no grupo das que tiveram acesso ao atendimento de prénatal, um serviço disponível no Sistema Único de Saúde que vem aumentando a sua cobertura no Brasil.

Sobre o questionamento da realização do teste sorológico para Hepatite B durante o pré-natal, 21 $(52,5 \%)$ puérperas afirmaram ter feito o exame, enquanto 19 (47,5\%) disseram não ter realizado.

A Hepatite B é descrita como passível de intervenção positiva em relação à gestante e ao recém-nascido; sendo prioridade a sua prevenção, o diagnóstico precoce durante a gestação e o cuidado com o feto de mães diagnosticadas levando-se em conta a sua prevalência, as coberturas vacinais heterogêneas no Brasil, assim, reduzindo sobremaneira a transmissão vertical do $\mathrm{VHB}^{16}$.

A triagem laboratorial do VHB visa conhecer o status sorológico da mãe e reduzir o risco de transmissão vertical durante a gestação. Os exames devem ser realizados na primeira consulta do pré-natal, independentemente da idade gestacional, com a garantia de resultados, em no máximo 15 dias, para os devidos encaminhamentos ${ }^{6}$.

As puérperas também foram questionadas quanto ao recebimento de orientações sobre hepatite de forma geral no pré-natal. Ao total, 26 (65\%) relaram não ter recebido nenhum tipo de informação sobre o assunto, enquanto 14 (35\%) afirmaram ter recebido algum tipo de informação. Essa informação relata o quão preocupante é a situação, devido principalmente ao fato de que o profissional de saúde não promove o aconselhamento quando solicita o exame à gestante.

\section{Realização do teste para Hepatite B no pré-natal}

São abordados nesta etapa os resultados quantitativos e os DSC construídos a partir das ideias centrais.

Em relação ao número de puérperas que responderam ao questionamento da realização, 17 (44,74\%) mulheres relataram que durante as consultas de pré-natal não ter sido ofertado para as mesmas o teste sorológico 
para a Hepatite B pelo profissional de saúde, 18 (47,37\%) tiveram a oferta do teste para o VHB, mas o profissional não o explicou, ou seja, não promoveu aconselhamento e 3 (7,89\%), perfazendo a minoria, receberam algum tipo de informação quando o exame para o VHB foi solicitado.

A partir dos resultados encontrados é notório que mesmo a Hepatite B sendo uma doença reconhecida atualmente como um problema de saúde pública e seu controle epidemiológico para reduzir os números de pessoas infectadas baseia-se principalmente na prevenção de sua transmissão, ainda não há por parte dos profissionais de saúde que trabalham com o atendimento de pré-natal manejo adequado quanto à oferta do teste para o VHB. Observa-se que: quando o profissional de saúde oferta este exame ele não explica sua importância e as possíveis consequências do resultado para a gestante ou simplesmente não oferece o teste, já que a minoria ofertou o teste e explicou algo sobre o assunto para as puérperas entrevistadas neste estudo.

$\mathrm{O}$ primeiro discurso aborda as puérperas que não tiveram a oferta do teste sorológico para hepatite B no pré-natal pelo profissional de saúde. Durante as consultas de pré-natal são preconizados alguns exames de sorologia importantes para detecção de doenças na mãe possíveis de transmissão para o bebê, dentre outros exames de rotina. Quando o profissional de saúde solicita esses exames para gestante, principalmente os que estão ligados à positividade sorológica para afecções de transmissão vertical, este deve aconselhar a puérpera sobre o teste, explicando para o que serve e seus possíveis achados.

\section{Sem teste sorológico para Hepatite B}

Ninguém falou nada. Em nenhum momento foi solicitado, se fez eles não falaram comigo. Só sei que fiz todos os exames. Ela me dava os papeizinhos e eu vinha aqui no laboratório e eles colhiam o sangue. Foram tantas tiradas de sangue que eu nem lembro se foi feito. Ele pedia para fazer o exame, e falava que estava tudo normal. Eu fiz tantos exames que eu não sei. (DSC1)

Entretanto, segundo o primeiro discurso, compreende-se que o profissional, que atende às gestantes, solicita os exames laboratoriais do pré-natal como parte de uma rotina diária de trabalho, de caráter burocrático em muitas situações, sem compreender a importância de tais exames para a saúde maternoinfantil. Assim, para que ocorra a prevenção da cadeia de transmissão vertical da Hepatite B, se faz necessário um profissional de saúde capacitado que transmita informação para a gestante de forma dialógica, respeitando os seus direitos e mostrando-lhe possibilidades para lidar com as diversas situações existentes.

\section{Com teste sorológico para Hepatite B}

A ginecologista pediu. Ela explicou que evita vários tipos de doenças, e você pode tratar ainda com o neném na barriga. Eu lembro que ela me explicou que até no caso depois que o neném nasce [...] falou que depois do parto eu teria que tomar a vacina. Porque se no caso de outra gestação poderia ocorrer algum problema. (DSC 02)

Ao analisar o segundo discurso, referente às puérperas que tiveram a oferta do teste percebe-se o quão relevante é a informação fornecida pelo profissional à gestante durante o pré-natal, qualquer que seja o conteúdo abordado. A gestante tem a possibilidade de conhecer mais sobre si mesma, sobre os riscos a que se expõe e a forma de prevenção de doenças de transmissão vertical, neste caso a Hepatite B. Além de permitir que a mulher tire dúvidas relacionadas à temática e também seja uma agente multiplicadora de informação dentro do seu contexto comunitário.

Quando as puérperas foram questionadas sobre os saberes que tinham em relação ao teste sorológico para a Hepatite B, 33 (80,49\%) disseram não ter nenhuma informação sobre o assunto e $8(19,51 \%)$ descreveram algo referente ao teste para o VHB. Estes dados indicam que durante o pré-natal o profissional de saúde ao ofertar o exame para o VHB não abordou o assunto com a gestante ou a forma que apresentou a temática não foi adequada para o seu entendimento.

Independente do assunto a ser abordado, o prénatal com qualidade destaca-se como sendo o primeiro alvo a ser atingido quando se busca reduzir as taxas de morbimortalidade materna e perinatal e, para tanto, a viabilização dos programas exige atuação profissional competente e atualizada de modo contínuo ${ }^{17}$.

\section{Falta de conhecimento sobre Hepatite B}

Sei quase nada, não lembro. Já ouvi falar da hepatite, mas não assim detalhado. Ela não me falou nada. Quando você vai ter o neném, aí você fica imune na hora do corte, é tétano. [...] é tipo falta de sangue, anemia, [...] para saber se o sangue esta de acordo com o meu e com o do neném. (DSC3)

Quando as puérperas foram questionadas quanto ao conhecimento que detinham sobre o teste sorológico para Hepatite B a maioria disse não saber nada referente à temática. No terceiro discurso compreendese que há conceitos errôneos quanto ao assunto, como confundir o teste para o VHB com a vacina contra o tétano, anemia ou compatibilidade sanguínea.

\section{Conhecimento sobre Hepatite B}

Porque você tem que fazer se você não tiver tomado a vacina. $[. .$.$] para prevenir tanto a mãe como a criança.$ Já vi pela televisão que a gente pega pelo alicate. Que pode pegar entre relação sexual também. Eu sei que é uma doença que precisa ser detectada porque éperigosa na gestação pode passar para a criança [...]. (DSC4)

No quarto discurso são descritos pelas puérperas informações pertinentes à Hepatite B. Hoje em dia há pelo Ministério da Saúde campanhas a nível nacional de prevenção da transmissão da Hepatite B, principal- 
mente vinculados a mídia (televisão e internet). Mas é durante o pré-natal que há a possibilidade de abordar o tema com a gestante e seu parceiro de forma mais específica, além de ser uma forma de rastreamento de casos de sorologia positiva para a Hepatite B, ou seja, diagnóstico precoce na gestante e tratamento subsequente para gestante e recém-nascido.

Dentro do nível de atenção básica encontramse as ações desenvolvidas nas unidades básicas de saúde e Programa Saúde da Família. Existindo como competência para os profissionais que atuam nesta esfera de atenção: a promoção à saúde, a prevenção, a triagem sorológica e o acompanhamento de pacientes. Em relação à prevenção da transmissão vertical de Hepatite B, são descritas duas ações importantes, como: a primeira dose da vacina anti-hepatite $\mathrm{B}$ que deve ser aplicada após o nascimento, nas primeiras 12 horas de vida e em recém-nascidos de mãe sabidamente portadora do VHB a administração também de imunoglobulina humana anti-hepatite tipo $\mathrm{B}^{18}$.

Pode-se observar que em relação às vivências das puérperas sobre a realização do teste sorológico para a Hepatite B durante o pré-natal, 36 (87,8\%) disseram não ter vivência sobre o assunto, enquanto 5 (12,2\%) relaram ter alguma vivência.

Após análise em base de dados em saúde foi possível, através da busca por artigos sobre a temática transmissão vertical sobre Hepatite $B$, perceber que a maioria dos trabalhos publicados fala sobre a prevalência das gestantes em relação ao rastreamento sorológico da Hepatite B e o tratamento subsequente se o resultado for positivo. Porém, não há relatos sobre o conhecimento ou vivência das gestantes sobre o assunto.

A Organização Mundial de Saúde justifica a realização de testes de rotina para a detecção da Hepatite B na gestação, devido: a gravidade do problema representado pela doença, a disponibilidade de testes confiáveis, acessíveis e aceitáveis pela população, e a existência de meios preventivos de alta eficácia a serem aplicados naqueles diagnosticados como positivos $^{19}$.

\section{Vivência sobre teste da Hepatite B}

Na minha primeira gestação eu assisti palestra e fiz o exame. Deve ser para a proteção do bebê. No caso se prevenir na mãe, previne também na criança, porque antes de acontecer o pior eles tratam a mãe, para a criança futuramente não ter esse problema [...] eu acho muito interessante porque antigamente não tinha tanto recurso, hoje em dia já tem tudo, você pode detectar não só a Hepatite B[...]. (DSC5)

O último discurso expõe relatos de puérperas que vivenciaram situações durante o pré-natal referente ao teste de Hepatite B, ou seja, tiveram contato com alguma informação que as proporcionaram conheci- mentos importantes. Durante o pré-natal o profissional de saúde pode abordar temáticas relevantes para a gestante utilizando-se de estratégias diversas, seja através de atividades individuais ou em grupo, aprimorando técnicas de ensino-aprendizagem e usando veículos variados de transmissão de informação. $\mathrm{O}$ profissional deve sempre estar atento às demandas e necessidades das gestantes e seus contextos familiares e comunitários para intervir de forma ativa no processo saúde-doença, focando sempre os aspectos de promoção e prevenção da sua saúde.

\section{Conclusão}

A pesquisa possibilitou o alcance do objetivo proposto inicialmente. Quanto à identificação de como ocorreu à oferta do exame para o VHB durante o pré-natal, sabe-se que mesmo havendo a recomendação do Ministério da Saúde para a oferta e a realização do teste sorológico para Hepatite B durante o pré-natal há um déficit na forma como é abordado o tema com a mulher; ou, em muitos casos, em que o profissional de saúde apenas solicitou o exame, não explicando a importância do mesmo.

Referente ao conhecimento das puérperas sobre o teste sorológico para a Hepatite B observa-se que a minoria relatou algo pertinente sobre o assunto e o descreveu como sendo importante para prevenção da transmissão vertical da Hepatite B. Assim, percebese que quando o profissional de saúde informa e aconselha a gestante durante o pré-natal sobre algo referente a este exame ou qualquer assunto relacionado às hepatites virais, a mulher tem a possibilidade de reconhecer seus riscos e modificá-los frente aos resultados possíveis do teste para o VHB.

O papel do enfermeiro é primordial durante o pré-natal, pois é o responsável em prestar assistência de enfermagem à gestante e promover o controle das doenças transmissíveis, como a Hepatite B. É imprescindível durante as consultas do pré-natal, o enfermeiro abordar tantos os aspectos da oferta do teste, como das formas de prevenção e tratamento.

O estudo teve como limitação o fato da coleta de dados ter sido realizada em apenas uma maternidade. Sugere-se que em próximos estudos seja realizada a coleta de dados em mais de uma maternidade, para que a abrangência seja ampliada.

\section{REFERÊNCIAS}

1.Araújo TME, Silva NC. Hepatite B: prevalência de marcadores sorológicos em profissionais de enfermagem de emergência. Rev enferm UERJ. 2014; 22:834-9.

2.Miranda MMS, Souza LMG, Aguiar RALP, Correa MDC, Maia MMM, Borges RS, Melo VH. Rastreamento das infecções perinatais na gravidez: realizar ou não? Femina. 2012; 40:13-22. 
3.Araújo TME, Sá LC, Santos AS, Faustino SKM, Tapety FI. Prevalência da hepatite B em usuários do laboratório Central do Piauí. Rev enferm UERJ [Internet]. 2012 [citado em 5 out 2012]. 20; 229-34. Disponível em: http://www.facenf.uerj.br/v20n2/v20n2a15.pdf 4.Ministério da Saúde (Br). Hepatites virais: o Brasil está atento. Brasília (DF): Ministério da Saúde; 2008. 5.Ministério da Saúde (Br). Programa Nacional de DST e Aids. Prevalências e frequências relativas de Doenças Sexualmente Transmissíveis (DST) em populações selecionadas de seis capitais brasileiras. Brasília (DF): Ministério da Saúde; 2005.

6.Ministério da Saúde (Br). Departamento de Ações Programáticas Estratégicas. Pré-natal e Puerpério: atenção qualificada e humanizada manual técnico. Brasília (DF): Ministério da Saúde; 2006.

7.Arraes LC, Sampaio AS, Barreto SG, Agra MS, Lorenzato F. Prevalência de hepatite $B$ em parturientes e perfil sorológico perinatal. Rev Bras Gineco Obstet. 2003; 25:571-6. 8.Kupek E, Oliveira JF. Transmissão vertical do HIV, da sífilis e da hepatite B no município de maior incidência de AIDS no Brasil: um estudo populacional no período de 2002 a 2007. Rev Bras Epidemiol. 2012; 15:478-87. 9.Ferezin RI, Bertolini DA, Demarchi IG. Prevalência de sorologia positiva para HIV, hepatite B, toxoplasmose e rubéola em gestantes do noroeste paranaense. Rev Bras Ginecol Obstet. 2013; 35:66-70

10.Brasil. Presidência da República, Lei no 7498 , de 25 de junho de 1986. Dispõe sobre a regulamentação do exercício da enfermagem, e dá outras providências [Internet]. Brasília (DF); 1986. [citado em 05 out 2014]. Disponível em: http://www.jusbrasil.com.br/ legislacao/128195/lei-7498-86

11.Alves ACP, Figueiredo FER, Sousa NPL, Oliveira DR, Sousa WM. Aplicação da tecnologia leve no pré-natal: um enfoque na percepção das gestantes. Rev enferm UERJ. 2013; 21:648-53.

12.Pinheiro J, Zeitoune RCG. O profissional de enfermagem e a realização do teste sorológico para hepatite B. Rev enferm UERJ [Internet] 2009 [citado em 5 out 2014]. 17:30-4. Disponível em: http://www.facenf.uerj.br/v17n1/v17n1a06.pdf 13.Cardoso LSM, Mendes LL, Melendez GV. Diferenças na atenção pré-natal nas áreas urbanas e rurais do Brasil: estudo transversal de base populacional.Rev Min Enferm. 2013; 17:85-92.

14.Feitosa JA, Coriolano MW, Alencar EM, Lima LS. Aconselhamento do pré-teste anti-HIV no pré-natal: percepções da gestante. Rev enferm UERJ [Internet] 2010 [citado em 5 out 2014]. 18:559-64. Disponível em: http://www.facenf.uerj.br/v18n4/v18n4a10.pdf

15.Rocha R, Oliveira C, Silva DKF, Bonfim C. Mortalidade neonatal e evitabilidade: uma análise do perfil epidemiológico. Rev enferm UERJ [Internet] 2011 [citado em 5 out 2014]. 19:114-20. Disponível em: http://www. facenf.uerj.br/v19n1/v19n1a19.pdf

16.Ministério da Saúde (Br). Departamento de Ações Programáticas Estratégicas. Gestação de alto risco: manual técnico. Brasília (DF): Ministério da Saúde; 2010. 17.Costa GRC, Chein MBC, Gama MEA, Coelho LSC, Costa ASV, Cunha CLF, Brito LMO. Caracterização da cobertura do pré-natal no Estado do Maranhão. Rev Bras Enferm. 2010; 6:1005-9.

18.Ministério da Saúde (Br). Departamento de Vigilância Epidemiológica. Hepatites Virais: o Brasil está atento. Brasília (DF): ANVISA; 2005.

19.Perim EB, Passos ADC. Hepatite B em gestantes atendidas pelo Programa do Pré-natal da Secretaria Municipal de Saúde de Ribeirão Preto, Brasil: prevalência da infecção e cuidados prestados aos recém-nascidos. Rev Bras Epidemiol. 2005; 8:272-81. 